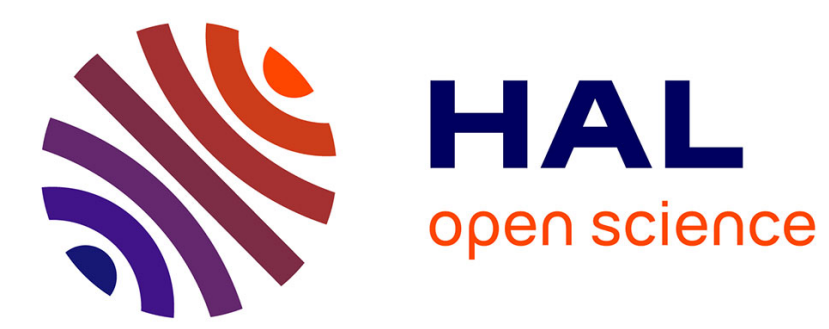

\title{
Cognitive biases and the representative agent
}

\author{
Elyès Jouini, Clotilde Napp
}

\section{To cite this version:}

Elyès Jouini, Clotilde Napp. Cognitive biases and the representative agent. 2009. halshs-00488570

\section{HAL Id: halshs-00488570 \\ https://shs.hal.science/halshs-00488570}

Preprint submitted on 2 Jun 2010

HAL is a multi-disciplinary open access archive for the deposit and dissemination of scientific research documents, whether they are published or not. The documents may come from teaching and research institutions in France or abroad, or from public or private research centers.
L'archive ouverte pluridisciplinaire HAL, est destinée au dépôt et à la diffusion de documents scientifiques de niveau recherche, publiés ou non, émanant des établissements d'enseignement et de recherche français ou étrangers, des laboratoires publics ou privés. 


\title{
Cognitive biases and the representative agent
}

\author{
Elyès Jouini, Université Paris Dauphine. \\ Clotilde Napp, CNRS and Université Paris Dauphine.
}

November 24, 2009

\begin{abstract}
In this paper, we show that behavioral features can be obtained at a group level when the individuals of the group are heterogeneous enough. Starting from a standard model of Pareto optimal allocations, with expected utility maximizers and exponential discounting, but allowing for heterogeneity among individual beliefs and individual time preference rates, we show that the representative agent has an inverse S-shaped probability distortion function and hyperbolic discount rates. As an application of this result, we show that an agent with a probability weighting function as in Cumulative Prospect Theory (resp. an ambiguity averse agent, resp. an hyperbolic discounting agent) may be represented as a collection of agents with noisy beliefs (resp. heterogeneous beliefs, resp. heterogeneous constant discount rates).

JEL Codes : G11; D81; D84; D87; D03; H43

Keywords: behavioral agent, hyperbolic discounting, probability weighting function, representative agent, neurofinance, ambiguity aversion.
\end{abstract}

\section{Introduction}

In this paper, we analyze a model of Pareto optimal allocations with von Neuman Morgenstern utility maximizing agents and exponential discounting. Agents are heterogeneous, in the sense

\footnotetext{
${ }^{*}$ Corresponding author: jouini@ceremade.dauphine.fr, +33144054226
} 
that they might differ in their beliefs and in their time preference rates. At the aggregate level, the social welfare function of this economy is characterized by a social/representative belief and a social/representative time preference rate. We examine these social characteristics and we show that we retrieve, at the aggregate level, behavioral properties that have been proved to be true at the individual level in recent literature. The group acts as a behavioral agent and these behavioral properties at the aggregate level are generated by heterogeneity alone.

We start by introducing natural notions of optimism and pessimism and we assume that beliefs are heterogeneous enough in order to allow for optimistic as well as pessimistic agents in the initial set of von Neuman Morgenstern utility maximizing agents. In such a setting, we obtain that the representative agent can neither be everywhere optimistic nor everywhere pessimistic; she is optimistic for the good states of the world and pessimistic for the bad states of the world. As in the SP/A Theory of Lopes (1987), the representative agent behaves as if she had fear (need for security) for very bad events and hope (desire for potential) for very good events. The representative agent puts more weight on extreme events. We show that the distribution of outcomes from the representative agent point of view is portfolio dominated by the objective distribution. This means that heterogeneity generates doubt at the aggregate level. This effect is reinforced when agents are more risk tolerant or when there is more heterogeneity among agents.

The representative agent distorts the objective distribution of aggregate endowment. We analyze this distortion and we show that the distortion function (defined as the transformation of the objective decumulative distribution function into the decumulative distribution function of the representative agent) is inverse S-shaped as is the probability weighting function in Cumulative Prospect Theory. We show that we are able to fit relatively well standard probability weighting functions of the Cumulative Prospect Theory literature (Tversky and Kahneman, 1992, Tversky and Fox, 1995, Prelec, 1998, among others). We analyze how the distribution of individual characteristics in the group governs the shape of the resulting representative agent 
distortion function. Following Gonzalez and Wu (1999) terminology, we show that attractiveness at the aggregate level is directly related to the average level of optimism while discriminability is related to beliefs heterogeneity.

As far as discount rates are concerned, we obtain that the aggregate time preference rate is decreasing, which is consistent with "hyperbolic discounting". It converges to the time preference rate of the most patient individual. These properties are similar to those obtained in a deterministic setting by Gollier-Zeckhauser (2005) and Lengwiler (2005) but are derived here in a stochastic setting that takes into account beliefs heterogeneity.

As a main application of these results we obtain that a behavioral individual (i.e. an individual whose preferences are governed by hyperbolic discounting and who distorts the distributions through a probability weighting function) behaves as would a group of standard heterogeneous vNM individuals with exponential discounting. Our results can then be related to Neuroeconomics. As underlined by Cohen (2005), findings from neuroscience "provide support for a view of the brain as a confederation of systems and behavior as the outcome of an interaction among these, (...) when disagreements arise, behavior reflects the outcome of a competition among systems". We show that a model of the brain as a central planner who, for the evaluation of a given prospect, maximizes the social welfare of a collection of competing doers (mental processes with heterogeneous time preference rates and noisy beliefs about the prospect under consideration) leads to hyperbolic discounting and to probability weighting functions that have the same shape as in the Cumulative Prospect Theory (CPT). Furthermore, such a model of individual behavior accomodates with a distinction between risk and uncertainty and exhibits, for some values of the parameters, ambiguity aversion. In such a framework, ambiguity aversion, inverse S-shaped probability weighting functions and hyperbolic discounting result from doers heterogeneity.

Note that we don't pretend to retrieve all features of CPT on the aggregate belief nor all features of the time preference rate as in e.g. Loewenstein and Prelec (1992). We only retrieve 
one of the three main features of CPT, namely the inverse S-Shaped probability distribution weighting function (the other two being the presence of a reference point and the presence of loss aversion). This comes from the fact that we have introduced heterogeneity on the beliefs only, hence the behavioral property that we retrieve deals with the belief only. We also only obtain the "hyperbolic" property of the time preference rate and not other behavioral properties such as the different (discounting) treatment of gains and losses.

The paper is organised as follows. Section 2 presents the model. Section 3 analyses the properties of the belief of the representative agent, while Section 4 analyses the properties of the time preference rate of the representative agent. Section 5 provides possible applications.

\section{$2 \quad$ The Setting}

We consider an economy with a single consumption good and with agents who have the same utility function but heterogeneous beliefs. Aggregate endowment in the consumption good is described by a random variable $e^{*}$ defined on a probability space $(\Omega, F, P)$. We let $I$ denote the set of heterogeneous agents. We assume that the common utility function is CRRA with

derivative given by $u^{\prime}(x)=x^{-\frac{1}{\eta}}$. Each agent has a subjective belief $Q_{i}$ and wants to maximize her von Neumann Morgenstern utility for consumption of the form $U_{i}(c)=E^{Q_{i}}[u(c)]$. We let $M^{i}$ denote the density of $Q_{i}$ with respect to the probability $P$, hence agent $i$ 's utility for consumption can equivalently be written in the form $U_{i}(c)=E\left[M^{i} u(c)\right]$.

In such an economy, we consider the aggregate utility function $U$ defined as the solution of the following maximization program

$$
U\left(e^{*}\right) \equiv \max _{\sum_{i \in I} y^{i}=e^{*}} \sum_{i \in I} \lambda_{i} E\left[M^{i} u\left(y^{i}\right)\right]
$$

where $\left(\lambda_{i}\right)$ are given positive weights. The aggregate utility function corresponds to the value of the social welfare function at the Pareto optimum when agent $i$ is granted a weight $\lambda_{i}$ by a 
social planner. The index $i$ may also represent a group of agents with common beliefs $M^{i}$ and $y^{i}$ then represents the total consumption of the group and $\lambda_{i}$ the sum of the weights granted by the social planner to the individuals in the group. When the social planner grants the same weight to all the agents in the economy, the weight $\lambda_{i}$ represents the proportion of agents that have the same belief $M^{i}$. From a social planner point of view, the aggregate utility function corresponds to the highest social utility level among all possible endowment distributions across agents.

The number of agents can be finite or infinite. In the case of an infinite number of agents, sums are replaced by integrals. We obtain the following representation result.

\section{Proposition 1 Representative Agent}

The aggregate utility for consumption is given by

$$
U\left(e^{*}\right)=E\left[M u\left(e^{*}\right)\right]
$$

with

$$
M=\left(\sum_{i \in I} \gamma_{i}\left(M^{i}\right)^{\eta}\right)^{\frac{1}{\eta}}
$$

for $\gamma_{i}=\lambda_{i}^{\eta}$. The representative agent belief is then given by $M=\left(\sum_{i \in I} \gamma_{i}\left(M^{i}\right)^{\eta}\right)^{\frac{1}{\eta}}$.

This means that, at the Pareto optimum, the aggregate utility is given by the utility of a representative agent endowed with an average belief (and the same utility function as each of the agents). In particular, if all the agents share the same belief, then the representative agent will share this common belief. If we think of $e^{*}$ as a given prospect for the group $I$ of agents, the aggregate utility $U\left(e^{*}\right)$ corresponds to the social welfare associated with the optimal allocation of $e^{*}$ across the members of the group and is given by the utility of the representative agent.

In the same way as the representative agent belief $M$ is an average of the individual beliefs $M^{i}$, we show in the next corollary that the density $f^{M}$ of $e^{*}$ for the representative agent is an 
average of the densities $f^{i}$ of $e^{*}$ for the different agents. For this purpose, let us assume that for all $i \in I$, the distribution of $e^{*}$ for agent $i$ admits a density ${ }^{1}$ (with respect to the Lebesgue measure on the real line), denoted by $f^{i}$. We also assume that the distribution of $e^{*}$ under the probability $P$ admits a density and we denote it by $f$. Since we don't have $E[M]=1$ (except in the specific logarithmic utility setting) we need the following technical definition. We say that the distribution of a random variable $X$ admits a "density $f_{X}$ for the representative agent" if for all function $h$, we have $E[M h(X)]=\int h(x) f_{X}(x) d x$. Moreover, in order to analyse the relative weights of the different states of the world from the representative agent point of view, we introduce the probability measure $Q$ defined by $\frac{d Q}{d P} \equiv \frac{M}{E[M]}$.

Corollary 2 The distribution of $e^{*}$ admits the following density for the representative agent

$$
f^{M}=\left(\sum_{i \in I} \gamma_{i}\left(f^{i}\right)^{\eta}\right)^{1 / \eta}
$$

which is a power average of the initial densities. In particular, for $\eta=1$, the distribution of $e^{*}$ for the representative agent is a mixture of the individual subjective distributions.

As an immediate consequence of Corollary 2, we get that for any measurable real-valued function $\varphi$, the distribution of $\varphi\left(e^{*}\right)$ admits the density $f^{M, \varphi}=\left(\sum_{i \in I} \gamma_{i}\left(f^{i, \varphi}\right)^{\eta}\right)^{1 / \eta}$ for the representative agent where $f^{i, \varphi}$ denotes the density of the distribution of $\varphi\left(e^{*}\right)$ for agent $i$. This implies in particular that in the case $\eta=1$, if each agent anticipates a normal distribution on $\log e^{*}$, then the distribution of $\log e^{*}$ is a mixture of normal distributions.

\footnotetext{
${ }^{1}$ In other words, the distribution of $e^{*}$ under $Q_{i}$ is absolutely continuous with respect to the Lebesgue measure.
} 


\section{Behavioral properties of the group}

\subsection{Qualitative properties}

The next two simple examples illustrate the qualitative properties of the endowment distribution from the representative agent point of view. The proofs can be found in the Appendix.

Example 1. Let us assume that all utility functions are logarithmic $(\eta=1)$. We have

$$
E^{Q}\left[e^{*}\right]=\sum_{i \in I} \gamma_{i} E^{Q_{i}}\left[e^{*}\right]
$$

which means that the mean at the aggregate level is given by an arithmetic average of the individual means. The variance is given by

$$
\operatorname{Var}^{Q}\left[e^{*}\right]=\sum_{i \in I} \gamma_{i} \operatorname{Var}^{Q_{i}}\left[e^{*}\right]+\operatorname{Var}_{i}\left(E^{Q_{i}}\left[e^{*}\right]\right)
$$

where $\operatorname{Var}_{i}\left(E^{Q_{i}}\left[e^{*}\right]\right) \equiv \sum_{i \in I} \gamma_{i}\left(E^{Q_{i}}\left[e^{*}\right]\right)^{2}-\left(\sum_{i \in I} \gamma_{i} E^{Q_{i}}\left[e^{*}\right]\right)^{2}$ measures beliefs (on the mean) heterogeneity. This means that the variance at the aggregate level is given not only by an arithmetic average of the individual variances, but also by an additional term related to beliefs dispersion. The variance is "increased" at the aggregate level and this increase is proportional to the level of beliefs heterogeneity: beliefs heterogeneity generates "doubt".

Example 2. Let us assume that the objective distribution of aggregate endowment is lognormal with $e^{*} \sim_{P} \ln \mathcal{N}\left(\mu, \sigma^{2}\right)$ and that we have two equally weighted groups of agents, both with lognormal subjective distributions for aggregate endowment, $e^{*} \sim_{Q_{i}} \ln \mathcal{N}\left(\mu_{i}, \sigma^{2}\right)$ for $i=1,2$. The distribution of $\log e^{*}$ for the representative agent is not Gaussian and when agents' beliefs are heterogeneous enough $\left(\left|\mu_{1}-\mu_{2}\right|>\frac{2 \sigma}{\sqrt{\eta}}\right)$, the distribution of $\log e^{*}$ is bimodal (see Figure 1). When $\mu=\frac{\mu_{1}+\mu_{2}}{2}$, the distribution of $\log e^{*}$ for the representative agent is Portfolio Dominated ${ }^{2}$

\footnotetext{
${ }^{2}$ Let us recall that a distribution $f$ dominates a distribution $g$ in the sense of Portfolio Dominance $\left(f \succeq_{P D} g\right)$ if we have $\int u^{\prime}(x)(x-a) f(x) d x=0 \Longrightarrow \int u^{\prime}(x)(x-a) g(x) d x=0$ for any real number $a$ and any non-decreasing concave function $u$. This concept has been introduced in the context of portfolio problems by Landsberger and Meilijson (1993) and further studied by Gollier (1997). It characterizes the changes in the distribution of the
} 
by the objective distribution. Hence, aggregate endowment $e^{*}$ is considered as more risky by the representative agent than it actually is. In particular, we have $\operatorname{Var}^{Q}\left[\log e^{*}\right]>\operatorname{Var}^{P}\left[\log e^{*}\right]$. This last property still holds for general $\left(\mu_{i}\right)$. Figures 1 and 2 illustrate these conclusions in different settings. Note that Figure 1 is similar to Figure 8.2 in Shefrin (2005). For $\eta>\eta^{\prime}$ and associated representative agent probability measures $Q^{\eta}$ and $Q^{\eta^{\prime}}$, we have $\frac{d Q^{\eta}}{d Q^{\eta^{\prime}}}=h_{\eta, \eta^{\prime}}\left(e^{*}\right)$ where $h_{\eta, \eta^{\prime}}$ is symmetric with respect to $\frac{\mu_{1}+\mu_{2}}{2}$, decreasing before $\frac{\mu_{1}+\mu_{2}}{2}$ and increasing after $\frac{\mu_{1}+\mu_{2}}{2}$. A higher level of risk tolerance induces then a portfolio dominated shift in the representative agent's distribution. In particular, $\operatorname{Var}^{Q}\left[\log e^{*}\right]$ increases with the level or risk tolerance $\eta$. The interpretation is the following. When there is heterogeneity, each agent consumes a larger proportion of aggregate endowment in states of the world that she considers more likely. This leads to heterogeneous allocations and generates variance at the aggregate level. However, this effect is counterbalanced by risk aversion. Consequently, the higher the level of risk tolerance, the more heterogeneous the members of the group are in their optimal allocations. Figure 2 illustrates this result.

For normal distributions $\mathcal{N}\left(\mu_{i}, \sigma^{2}\right)$, there is a natural order on the set of possible densities induced by the natural order on the means $\left(\mu_{i}\right)$. Agents with a larger (resp. smaller) $\mu_{i}$ can be referred to as more optimistic (resp. pessimistic). We generalize these notions of relative pessimism/optimism in the following definition. If we assume that $P$ is the objective probability, then we are also able to introduce absolute notions of pessimism/optimism.

Definition 1 For $i, j \in I$, agent $i$ is said to be more optimistic than agent $j$ and we denote it by $f_{i} \succcurlyeq_{\text {opt }} f_{j}$ if and only if $\frac{f_{i}}{f_{j}}$ is nondecreasing. The optimism relation $\succcurlyeq_{\text {opt }}$ is an order on the set $\left(f_{i}\right)_{i \in I}$. If $P$ is the objective probability, then agent $i$ is said to be optimistic (resp. pessimistic) if $\frac{f_{i}}{f}$ is nondecreasing (resp. nonincreasing).

Definition 1 can be rephrased in terms of Monotone Likelihood Ratio Dominance (MLR) ${ }^{3}$

returns of the risky asset that lead to an increase in demand for the risky asset irrespective of the risk-free rate. It is then related to the degree of riskiness. See also Jouini and Napp (2008).

${ }^{3}$ This concept is widely used in the statistical literature and was first introduced in the context of portfolio 
: agent $i$ is more optimistic than agent $j$ if the distribution of $e^{*}$ for agent $i$ (i.e., under $Q_{i}$ ) dominates the distribution of $e^{*}$ for agent $j$ (i.e., under $Q_{j}$ ) in the sense of the MLR. For a given agent $i$, if we let $g_{i}$ denote the transformation of the objective decumulative distribution function $F$ into the agent's subjective decumulative distribution function $F_{i}$, i.e. such that $F_{i}=g_{i} \circ F$, it is easy to check that $\frac{f_{i}}{f}$ is nondecreasing (resp. nonincreasing) if and only if $g_{i}$ is convex (resp. concave). This means that our concept of optimism/pessimism is the analog, in the expected utility framework, of the concept of optimism/pessimism introduced by Diecidue and Wakker (2001) in a RDEU framework. Other concepts of optimism/pessimism have been proposed in the literature. In particular, Yaari (1987), Chateauneuf and Cohen (1994) and Abel (2002) propose a definition based on First Stochastic Dominance ${ }^{4}$. Note that MLR dominance is stronger than FSD.

A MLR dominated shift for a given distribution reduces the mean and if $f_{i} \succcurlyeq_{\text {opt }} f_{j}$ then we have $E^{Q_{i}}\left[e^{*}\right] \geq E^{Q_{j}}\left[e^{*}\right]$. This last condition characterizes the MLR dominance when we restrict our attention to a family of lognormal distributions with the same variance parameter and we then retrieve that agent $i$ is more optimistic than agent $j$ if and only if $\mu_{i}>\mu_{j}$. In that case, optimistic (resp. pessimistic) agents are then characterized by $\mu_{i}>\mu$ (resp. $\mu_{i}<\mu$ ) as in Shefrin (2005).

Proposition 3 We suppose that there are at least one optimistic agent denoted by $f_{\text {opt }}$ and one pessimistic agent denoted by $f_{\text {pess }}$ in the set I of agents. We also assume that $\lim _{+\infty} \frac{f_{o p t}}{f}=$ $\lim _{-\infty} \frac{f_{p e s s}}{f}=+\infty$ and $\lim _{-\infty} \frac{f_{o p t}}{f}=\lim _{+\infty} \frac{f_{\text {pess }}}{f}=0$.

1. The representative agent can neither be optimistic, nor pessimistic, i.e. $\frac{f_{M}}{f}$ is non monotone.

2. The representative agent overestimates the weight of the "good states of the world" (high

problems by Landsberger and Meilijson (1990). More precisely, Landsberger and Meilijson (1990) showed that in the standard portfolio problem a MLR shift in the distribution of returns of the risky asset leads to an increase in demand for the risky asset for all agents with nondecreasing utilities.

${ }^{4}$ More precisely, in an expected utility framework Abel (2002) defines pessimism by the condition $F_{i} \geq F$ (First Stochastic Dominance) that corresponds to the condition $g_{i} \geq I d$ introduced by Chateauneuf and Cohen (1994) in a RDEU setting. 
values of $e^{*}$ ) as well as the weight of "bad states of the world" (low values of $e^{*}$ ), i.e. $f_{M}(x) \geq f(x)$ for $x \leq \underline{x}$ and $f_{M}(x) \geq f(x)$ for $x \geq \bar{x}$ where $\underline{x}$ and $\bar{x}$ are given real numbers.

3. If one of the agents denoted by $f_{\text {opt }}^{\max }$ is more optimistic than all the other agents and if one of the agents denoted by $f_{\text {pess }}^{\max }$ is more pessimistic than all the other agents, then the representative agent behaves like the most pessimistic individual for low values of $e^{*}$ and behaves like the most optimistic individual for high values of $e^{*}$, i.e. $f_{M} \sim_{+\infty} f_{\text {opt }}^{\max }$ and $f_{M} \sim_{-\infty} f_{\text {pess }}^{\max }$

By definition, $\frac{f_{o p t}}{f}$ (resp. $\frac{f_{\text {pess }}}{f}$ ) is nondecreasing (resp. nonincreasing). In Proposition 3, we slightly reinforce these conditions by further assuming that the values of $\frac{f_{o p t}}{f}$ (resp. $\frac{f_{p e s s}}{f}$ ) range from zero to infinity. Notice that these conditions are satisfied in the case of lognormal distributions.

It appears from this proposition that as long as there are optimistic as well as pessimistic agents in the set $I$ of agents, the representative agent behaves like the individual agents considered in the behavioral economics and/or psychology literature. Indeed, she puts more weight on small probability events with large consequences as in the Cumulative Prospect Theory of Kahneman and Tversky (1992). She has fear (need for security) for very bad events and hope (desire for potential) for very good events as in the SP/A Theory of Lopes (1987). Everything works then as if the representative agent distorted the objective distribution of $e^{*}$. In the next section, we analyze more precisely how this distortion operates.

\subsection{The distortion function}

Let us first recall that in the context of Cumulative Prospect Theory, the probability weighting function is defined as the function that transforms the decumulative objective distribution function into the decumulative subjective distribution function. This probability weighting function operates on any prospect. In our framework, we have seen that the representative 
agent distorts the objective distribution of $e^{*}$. In the present section, we will describe this distortion in terms of a transformation of the decumulative distribution function of $e^{*}$ and we will see that this distortion function has the same shape as the probability weighting function of the Cumulative Prospect Theory. We will then use the typology introduced by Gonzalez and $\mathrm{Wu}(1999)$ on the probability weighting functions to describe our distortion function and to relate its characteristics to the individual beliefs of the agents. In particular, we analyze how shifts in the distribution of individual beliefs impact the distortion function.

With the same notations as in Section 2, we denote by $g$ the distortion function that transforms the objective decumulative distribution function $\int_{x}^{\infty} f(s) d s$ into the decumulative distribution function of the group $\int_{x}^{\infty} f^{M}(s) d s$, i.e. such that $g\left(\int_{x}^{\infty} f(s) d s\right)=\int_{x}^{\infty} f^{M}(s) d s$. The next proposition assumes that there are at least one optimistic and one pessimistic agent in the set $I$ and characterizes the shape of $g$ under this assumption.

Proposition 4 1. In the lognormal setting with $\log e^{*} \sim_{Q_{i}} \mathcal{N}\left(\mu_{i}, \sigma^{2}\right)$ for $i=1, \ldots, N$ and if the set I is made of both optimistic and pessimistic agents then the function $g$ is inverse S-shaped: concave then convex.

2. In the general setting, if there are at least one optimistic agent $f_{\text {opt }}$ and one pessimistic agent $f_{\text {pess }}$ with $\lim _{+\infty} \frac{f_{o p t}}{f}=\lim _{-\infty} \frac{f_{\text {pess }}}{f}=+\infty$ and $\lim _{-\infty} \frac{f_{\text {opt }}}{f}=\lim _{+\infty} \frac{f_{\text {pess }}}{f}=0$ and if $g$ is continuously twice differentiable on $[0,1]$, then $g$ is concave for small probabilities, and convex for high probabilities.

The function $g$ has then the same shape as the probability weighting function of the Cumulative Prospect Theory. This is in particular illustrated in Figure 3. A variety of methods have been used to determine the shape of the probability weighting function. Tversky and Kahneman (1992), Fox and Tversky (1995) and Prelec (1998) among others specify parametric forms (respectively $\omega(p)=\frac{p^{\gamma}}{\left[p^{\gamma}+(1-p)^{\gamma}\right]^{1 / \gamma}}, \omega(p)=\frac{\delta p^{\gamma}}{\left[\delta p^{\gamma}+(1-p)^{\gamma}\right]^{1 / \gamma}}$ and $\omega(p)=\exp -\left(-\log p^{\gamma}\right)$ ) and estimate them through standard techniques. Figure 4 permits to show that with a well chosen 
distribution of agents' characteristics we obtain a distortion function that perfectly fits Prelec (1998)'s function. Wu and Gonzalez (1996, 1998) and Abdellaoui (2000) avoid the potential problems of parametric estimation and directly derive from experimental studies the shape of the probability weighting function at the aggregate or individual level. The results of all these studies are (mostly) consistent with an inverse S-shaped weighting function, concave for small probabilities, and convex for moderate and high probabilities.

In the lognormal setting, if we denote by $\delta_{i}$ the quantity $\delta_{i}=\frac{\mu_{i}-\mu}{\sigma}$, it is interesting to remark that the distortion function $g$ only depends on the $\delta_{i}$ s and on the relative proportions $\gamma_{i}$ s and is independent of $\mu$ and $\sigma$. In other words, the distortion function only depends on how much the agents deviate from the objective mean in terms of standard deviation.

\subsection{Discriminability, attractiveness and individual agents' beliefs}

Let us analyse how the main features for the shape of the representative agent's distortion function $g$, for a given aggregate endowment $e^{*}$, are related to the individual characteristics of the agents in $I$. Gonzalez and Wu (1999) exhibit two main features for the shape of the probability weighting function in the context of CPT: diminishing sensitivity and attractiveness.

Attractiveness characterizes the absolute level of the function. Indeed, an inverse S-shaped function can be completely below the identity line, can cross the identity line at some point or can be completely above the identity line. Betting on the chance domain is more attractive when the graph of the probability weighting function graph is more "elevated". The definition of attractiveness is expressed in terms of First Stochastic Dominance (FSD). The probability weighting function $g_{1}$ is more attractive than the probability weighting function $g_{2}$ when the subjective density $f_{1}$ dominates the subjective density $f_{2}$ in the sense of the FSD. In our setting, we will say that a (representative agent's) distortion function $g_{1}$ associated with a set $I_{1}$ of agents is more attractive than a (representative agent's) distortion function $g_{2}$ associated with a set $I_{2}$ of agents if $f_{I_{1}}^{M}$ dominates $f_{I_{2}}^{M}$ in the sense of the FSD. Attractiveness of the distortion function 
is related to the level of optimism of the representative agent. In particular, since FSD is weaker than MLR, a more optimistic representative agent is associated with a more attractive distortion function.

Let $\left(\gamma_{i}\right)$ and $\left(\gamma_{i}^{\prime}\right)$ denote two possible distributions of agents' density functions. If the set $\left(f_{i}\right)_{i \in I}$ of agents' density functions is totally ordered with respect to the FSD order, we will say that the distribution $\left(\gamma_{i}^{\prime}\right)$ dominates the distribution $\left(\gamma_{i}\right)$ in the sense of the FSD if for any increasing family $\left(f_{i}\right)$, we have $\sum \gamma_{i}^{\prime} f_{i} \succcurlyeq_{F S D} \sum \gamma_{i} f_{i}$. In other words, the distribution $\left(\gamma_{i}^{\prime}\right)$ puts more weight on more attractive distributions. If the set $\left(f_{i}\right)_{i \in I}$ of agents' density functions is totally ordered with respect to the optimism order $\succcurlyeq_{\text {opt }}$, we will say that the distribution $\left(\gamma_{i}^{\prime}\right)$ dominates the distribution $\left(\gamma_{i}\right)$ in the sense of the MLR if whenever $f_{i} \succcurlyeq_{o p t} f_{j}$ we have $\frac{\gamma_{i}^{\prime}}{\gamma_{i}} \geq \frac{\gamma_{j}^{\prime}}{\gamma_{j}}$. In other words the ratio between the two densities $\left(\gamma_{i}^{\prime}\right)$ and $\left(\gamma_{i}\right)$ increases with agents' optimism and, in particular, the distribution $\left(\gamma_{i}^{\prime}\right)$ puts more weight on more optimistic agents.

In the next proposition we analyze the impact of shifts in the distribution of agents characteristics on the attractiveness of the distortion function and on the level of optimism of the representative agent.

Proposition 5 1. For log-utility functions and in the case of lognormal distributions $\log e^{*} \sim Q_{Q_{i}}$ $\mathcal{N}\left(\mu_{i}, \sigma^{2}\right)$ for $i=1, \ldots, N$, with the same variance parameter $\sigma^{2}$, a FSD shift in the distribution of the means $\left(\mu_{i}\right)$ increases attractiveness of the representative agent's distortion function.

2. For log-utility functions and general distributions, if the set $\left(f_{i}\right)_{i \in I}$ of agents' density functions is totally ordered with respect to the FSD order then a FSD shift in the distribution of agents' density functions increases attractiveness of the representative agent's distortion function.

3. For general CARA utility functions and general distributions, if the set $\left(f_{i}\right)_{i \in I}$ of agents' density functions is totally ordered with respect to the optimism order $\succcurlyeq_{\text {opt }}$ then a MLR 
dominated shift in the distribution of agents' density functions increases attractiveness of the representative agent's distortion function and the level of pessimism of the representative agent.

When all agents have logarithmic utility functions, attractiveness at the representative agent level increases with the weight granted to the more attractive density functions. Since FSD is weaker than MLR, attractiveness at the representative agent level increases with the weight granted to the more optimistic agents. This is illustrated by Figure 5. As shown in Proposition 5 , this last property can be extended to power utility functions if we replace FSD shifts on the distribution of agents' density functions by MLR shifts.

Diminishing sensitivity corresponds to the fact that people become less sensitive to changes in probability as they move away from a reference point. In the probability domain, the two endpoints 0 (certainly will not happen) and 1 (certainly will happen) serve as reference points and under this principle, increments near the endpoints of probability loom larger than increments near the middle of the scale. This concept is related to the concept of discriminability in psychophysics literature and can be illustrated by two extreme cases: a function that approaches a step function and a function that is almost linear.

In our setting we say that a representative agent's distortion function $g_{1}$ associated with a set $I_{1}$ of agents exhibits more discriminability than a representative agent's distortion function $g_{2}$ associated with a set $I_{2}$ of agents if there exists $x^{*} \in[0,1]$ such that $g_{1} \leq g_{2}$ for $x \leq x^{*}$ and $g_{1} \geq g_{2}$ for $x \geq x^{*}$. In the next proposition we show that the level of discriminability of the representative agent's distortion function is closely related to the level of disagreement among agents.

Let us consider as above a family of agents with lognormal distributions $\ln \mathcal{N}\left(\mu_{i}, \sigma^{2}\right)$. We denote by $\left(\mu_{i}\right)$ the support of the distribution of the mean parameter and by $\left(\gamma_{i}\right)$ the associated weights. Recall that a mean preserving spread is defined as a modification of the distribution set $\left(\gamma_{i}\right)$ on a set of three locations $\mu_{1}<\mu_{2} \lesseqgtr \mu_{4}$ with associated increments $\delta_{1} \geq 0, \delta_{2} \leq 0$ 
and $\delta_{3} \geq 0$ such that $\sum_{i=1}^{3} \delta_{i}=0$ and $\sum_{i=1}^{3} \delta_{i} \mu_{i}=0$. A mean preserving spread will be said symmetric if $\delta_{1}=\delta_{3}$.

Proposition 6 For log-utility functions and in the case of lognormal distributions $\log e^{*} \sim_{Q_{i}}$ $\mathcal{N}\left(\mu_{i}, \sigma^{2}\right)$, a symmetric mean-preserving spread on the distribution of the means $\left(\mu_{i}\right)$ decreases discriminability of the representative agent's distortion function.

Intuitively, this proposition means that when the level of disagreement among agents increases, then the representative agent focuses more on the endpoints of the probability domain and is less sensitive to probability variations in the middle of the scale. Figure 6 illustrates this result. It shows, in the setting with two agents, that discriminability decreases with the level of disagreement. When both agents agree on the objective distribution, the probability weighting function is linear. When the agents disagree, one of them overestimating the average payoff by twice the standard deviation and the other underestimating it by twice the standard deviation, we obtain a function that approaches a step function.

\section{The Setting with Heterogeneous Time Preference Rates}

In this section, we extend our framework in order to take into account the impact of time and of heterogeneous time preference rates across the agents. Aggregate endowment at a given date $t$ is described by a random variable $e_{t}^{*}$. Agents have different time preference rates $\left(\rho_{i}\right)$ and different subjective beliefs $Q_{i}$. We let $M_{t}^{i}$ denote the density at date $t$ of $Q_{i}$ with respect to the objective probability $P$ and $D_{t}^{i} \equiv \exp \left(-\rho_{i} t\right)$ the discount factor of agent $i$ between date 0 and date $t$. As previously, we consider the aggregate utility function $U$ defined as the solution of the following maximization program

$$
U\left(e_{t}^{*}\right)=\max _{\sum_{i \in I} y_{t}^{i}=e_{t}^{*}} \sum_{i \in I} \lambda_{i} E\left[M_{t}^{i} D_{t}^{i} u\left(y_{t}^{i}\right)\right]
$$


where $\left(\lambda_{i}\right)$ are given positive weights. Each agent is then characterized by a belief $M_{t}^{i}$, a discount factor $D_{t}^{i}$ and a weight $\lambda_{i}$.

We will say that the characteristics $\left(M_{t}^{i}, D_{t}^{i}, \lambda_{i}\right)_{i \in I}$ are independent if for almost all states of the world $\omega, M_{t}^{i}(\omega), D_{t}^{i}$ and $\lambda_{i}$ are independent ${ }^{5}$ as random variables on $I$. This property will be, in particular, satisfied when $I$ can be written in the form $I=J \times K \times L$ and when there exist characteristics $\left(\bar{M}_{t}^{j}\right)_{j \in J},\left(\bar{D}_{t}^{k}\right)_{k \in K}$ and $\left(\bar{\lambda}_{\ell}\right)_{\ell \in L}$ such that for $i=(j, k, \ell)$ we have $\left(M_{t}^{i}, D_{t}^{i}, \lambda_{i}\right)=\left(\bar{M}_{t}^{j}, \bar{D}_{t}^{k}, \bar{\lambda}_{\ell}\right)$. Roughly speaking, this property means that there is no specific correlation between beliefs and time preferences and that the weights granted by the social planner to the individuals in the economy are independent of their time and belief characteristics.

This condition is, in particular, satisfied when beliefs and time preferences are independent and when the agents are uniformly weighted in the social welfare function. This is also the case when the agents' weights are given by their relative wealth and when wealth, beliefs and time preferences are independent.

Assuming uniform weights is quite reasonable since there is no particular reason for the social planner to favor one agent with respect to another agent. The independence of beliefs and time preference rates is more disputable. They may be positively as well as negatively correlated, the independence condition may then be analyzed as a central scenario.

We easily obtain the following analog of Proposition 1 in the framework with heterogeneous time preference rates.

Proposition 7 If the characteristics $\left(M_{t}^{i}, D_{t}^{i}, \lambda_{i}\right)_{i \in I}$ are independent, then the aggregate utility for consumption is given by

$$
U\left(e_{t}^{*}\right)=E\left[M_{t} D_{t} u\left(e_{t}^{*}\right)\right]
$$

\footnotetext{
${ }^{5}$ More precisely, for any real valued (measurable) functions $f, g, h$ defined on the real line, we have $\frac{1}{|I|} \sum_{i \in I} f\left(M_{t}^{i}\right) g\left(D_{t}^{i}\right) h\left(\lambda_{i}\right)=\left(\frac{1}{|I|} \sum_{i \in I} f\left(M_{t}^{i}\right)\right)\left(\frac{1}{|I|} \sum_{i \in I} g\left(D_{t}^{i}\right)\right)\left(\frac{1}{|I|} \sum_{i \in I} h\left(\lambda_{i}\right)\right)$ a.e.
} 
with

$$
M_{t}=\left(\frac{1}{|I|} \sum_{i \in I}\left(M_{t}^{i}\right)^{\eta}\right)^{\frac{1}{\eta}} \text { and } D_{t}=\left(\frac{1}{|I|} \sum_{i \in I}\left(D_{t}^{i}\right)^{\eta}\right)^{\frac{1}{\eta}}
$$

The representative agent belief is then given by $M_{t}=\left(\frac{1}{|T|} \sum_{i \in I}\left(M_{t}^{i}\right)^{\eta}\right)^{\frac{1}{\eta}}$ and the representative agent time discount factor is given by $D_{t}=\left(\frac{1}{|I|} \sum_{i \in I}\left(D_{t}^{i}\right)^{\eta}\right)^{\frac{1}{\eta}}$.

This means that all the properties established in the previous section on the belief of the representative agent remain valid.

The properties of the representative agent time preference rate are easy to obtain. Note that the properties of a "consensus" time preference rate when there is heterogeneity on the individual time preference rates (and not on the beliefs) have already been studied in varying contexts. Indeed, the problem of the aggregation of the utility discount rates has been studied by Reinschmidt (2002) through a certainty equivalent approach, by Gollier and Zeckhauser (2005) and Nocetti and al. (2008) through a Benthamite/Pareto optimal approach, and by Lengwiler (2005) through an equilibrium approach. All these papers adopt a deterministic setting with no divergence on the beliefs of the agents. On the contrary our aim here is to derive the properties at the aggregate level simultaneously on the beliefs and on the time preference rate (and in a quite general stochastic setting).

We know that the representative agent time discount factor is given by $D_{t}=\left(\sum_{i \in I} \frac{1}{|I|}\left(D_{t}^{i}\right)^{\eta}\right)^{\frac{1}{\eta}}$ where $D_{t}^{i} \equiv \exp \left(-\rho_{i} t\right)$. We introduce the representative agent marginal time preference rate $\rho_{m}$ as well as the representative agent average time preference rate $\rho_{a}$, respectively defined by

$$
\rho_{m}^{D}(t) \equiv-\frac{D_{t}^{\prime}}{D_{t}} \text { and } \rho_{a}^{D}(t) \equiv-\frac{1}{t} \log D_{t}
$$

The average discount rate corresponds to the rate which, if applied constantly for all intervening years, would yield the discount factor $D_{t}$, whereas the marginal discount rate is the rate of change of the discount factor. It is easy to recover the average discount rate from the 
marginal discount rate since $\rho_{a}(t)=\frac{1}{t} \int_{0}^{t} \rho_{m}(s) d s$.

Let us state the following properties of the average and marginal time preference rates.

Proposition 8 Properties of the representative agent time preference rate

1. The representative agent average and marginal time preference rates are given by

$$
\begin{aligned}
& \rho_{a}^{D}(t)=-\frac{1}{t} \log \left[\frac{1}{N} \sum_{i=1}^{N} \exp \left(-\eta \rho_{i} t\right)\right]^{1 / \eta}, \\
& \rho_{m}^{D}(t)=\sum_{i=1}^{N} \frac{\exp \left(-\eta \rho_{i} t\right)}{\sum_{i=1}^{N} \exp \left(-\eta \rho_{i} t\right)} \rho_{i} .
\end{aligned}
$$

2. The representative agent time preference rates are lower than the average of the time preference rates, i.e.

$$
\rho_{m}^{D}(t) \leq \frac{1}{N} \sum_{i=1}^{N} \rho_{i}=\rho_{m}^{D}(0) \text { and } \rho_{a}^{D}(t) \leq \frac{1}{N} \sum_{i=1}^{N} \rho_{i}=\rho_{a}^{D}(0)
$$

with strict inequalities when $\rho_{i} \neq \rho_{j}$ for some $(i, j)$ in $I$.

3. "Behavioral Properties" : The representative agent time preference rates are decreasing with time. Moreover, the asymptotic discount rates are given by the lowest time preference rate, i.e. $\lim _{t \rightarrow+\infty} \rho_{a}^{D}(t)=\lim _{t \rightarrow+\infty} \rho_{m}^{D}(t)=\inf _{i}\left(\rho_{i}\right)$. The representative agent behaves for $t$ large enough like the most patient agent.

These formulas permit explicit computations for specific distributions of the individual time preference rates. For instance, if we assume a Gamma ${ }^{6}$ distribution $\gamma(\alpha, \beta)$ for the $\rho_{i} \mathrm{~s}$ we obtain

$$
\rho_{m}^{D}(t)=\frac{m^{2}}{m+\eta v^{2} t}
$$

\footnotetext{
${ }^{6}$ As mentioned in Section 2, sums should be replaced by integrals when dealing with continuous distributions. The density function of a gamma distribution $\gamma(\alpha, \beta)$ is given by $\frac{\beta^{\alpha}}{\Gamma(\alpha)} x^{\alpha-1} \exp (-\beta x)$. Its mean $m$ and its variance $v^{2}$ are respectively given by $m=\frac{\alpha}{\beta}$ and $v^{2}=\frac{\alpha}{\beta^{2}}$.
} 
where $m$ and $v^{2}$ respectively denote the mean and the variance of the considered distribution. It is immediate on this simple example that the marginal discount rate decreases with time and is hyperbolic as in Weitzman $(1998,2001)$. Furthermore, the speed of the decrease increases with the level of heterogeneity $v^{2}$ as well as with the level of risk tolerance.

The next proposition provides comparative statics results for shifts in the distribution $f_{\rho}$ of the individual time preference rates.

Proposition 9 1. A FSD (resp. SSD) dominated shift in the distribution $f_{\rho}$ of individual time preference rates decreases the representative agent average time preference rate $\rho_{a}^{D}$.

2. A MLR (resp. PD) dominated shift in the distribution $f_{\rho}$ of individual time preference rates decreases the representative agent marginal time preference rate $\rho_{m}^{D}$.

Second Stochastic Dominance as well as Portfolio Dominance are related to a notion of risk or of dispersion while First Stochastic Dominance and Monotone Likelihood Ratio Dominance are related to notions of shifts from low values to high values. Roughly speaking, Proposition 9 introduces the right concepts of dispersion and shifts and shows that more dispersion in agents' time preference rates as well as shifts to lower values of individual time preference rates decrease the representative agent's time preference rate.

\section{Applications}

We have seen that starting from a standard model with optimistic as well as pessimistic vNM and exponential discounting agents, we obtain, at the representative agent level, properties such as an inverse S-shaped distortion function and hyperbolic discounting, that are in line with recent empirical and experimental results. A possible interpretation of such a result is to consider that each individual subject to experiments behaves as a group of individuals at the equilibrium. This provides us with a possible representation of the brain as an organization with a social planner and heterogeneous doers, 19 Some doers are overoptimistic while others are 
overpessimistic. Similarly, some doers are impatient while others are more patient. Such an approach is in the same spirit as Brocas and Carillo (2008) where the authors divide the brain into two systems: an impulsive/myopic one and a cognitive/forward-looking one. However, while Brocas and Carillo (2008) model mainly relies on information asymmetries and principalagent models, our model relies on decentralization and optimal allocation approaches. Such a decomposition of the brain into different systems that are possibly in conflict are based on recent neuroscience and psychology evidences (see Cohen, 2005) related to intrapersonal tensions: temporal horizon conflicts, information conflicts (that may lead to information asymmetries as in Brocas and Carillo, 2008, but also to information diversity and beliefs heterogeneity as in our model) or utility evaluation conflicts. In economics, such decompositions have been first considered by Thaler and Shefrin (1981) and Shefrin and Thaler (1988).

\subsection{Noisy Beliefs and Cumulative Prospect Theory}

In this section we show that an individual who evaluates lotteries through the social welfare function associated with a collection of agents (neural systems or brain areas), each of them with specific noisy beliefs, is a CPT agent in the sense that she distorts the distribution of the lotteries through an inverse S-shaped weighting function (commpon to all lotteries).

We start by considereing normal distributions. Let us consider an individual who when facing a lottery whose payoff $x$ is described by a normal distribution $\mathcal{N}\left(\mu, \sigma^{2}\right)$ passes this information for evaluation to separate systems. Each system $i$ has a subjective belief $Q_{i}$ under which $x$ has a normal distribution $\mathcal{N}\left(\mu+\delta_{i} \sigma, \sigma^{2}\right)$. The parameter $\delta_{i}$ is fixed independently of $x$ and characterizes the system $i$. It might result from noise in the information transmission. In that case there is no specific reason for the average perceived signal to be biased and we should have $\sum \delta_{i}=0$. It might also result from a specialization of the different systems, some systems being optimistic, i.e., $\delta_{i}>0$ and others being pessimistic, i.e., $\delta_{i}<0$. We assume that the individual acts like a central planner looking for a Pareto optimal decomposition of the payoffs from the 
lottery among the systems and evaluates the lottery through the social welfare function, i.e.,

$$
U_{\delta}(x)=\max _{\sum x^{i}=x} \sum_{i} \gamma_{i} E^{Q_{i}}\left[u\left(x^{i}\right)\right]
$$

where the parameters $\gamma_{i}$ are the weights granted to the systems by the central planner or the distribution of the $\delta_{i} \mathrm{~s}$.

Proposition 10 Consider an individual who evaluates any lottery $x$ in the space $X$ of lotteries with normal payoffs through $U_{\delta}(x)$.

1. The individual is a CPT agent over the space $X$ in the sense that there exists a probability weighting function $g_{\delta}$ such that, for all lotteries $x$ in $X$ with density $f_{x}$ we have $U_{\delta}(x)=$ $\int f_{x, \delta}(s) u(s) d s$ where $g_{\delta}\left(\int_{t}^{\infty} f_{x}(s) d s\right)=\int_{t}^{\infty} f_{x, \delta}(s) d s$.

2. If there exist at least one optimistic and one pessimistic system, then $g_{\delta}$ is inverse $S$ shaped.

3. A MLR shift on the distribution of the $\delta_{i}$ s increases attractiveness of the probability weighting function $g_{\delta}$.

This means that a Pareto optimal decomposition leads to an overall (representative agent) evaluation that corresponds to the valuation that would be provided by a behavioral agent. The level of discriminability would then be directly related to the level of noise as illustrated in Proposition 6 in the case of log utility functions. The level of attractiveness would be associated to the level of systematic bias (if any) as a direct corollary of Proposition 5.

The behavior of the individual and the definition of the social welfare function $U_{\delta}$ can be naturally generalized to any lottery whose payoff is a function of a normal distribution. Indeed, consider a lottery whose payoff is of the form $v=\varphi(x)$ where $x$ is normally distributed as above 
and where $\varphi$ is a Borelian function. We may define $U_{\delta}(v)$ by

$$
U_{\delta}(v)=\max _{\sum v^{i}=v} \sum_{i} \gamma_{i} E^{Q_{i}}\left[u\left(v^{i}\right)\right]
$$

where the $Q_{i}$ s and the $\delta_{i}$ s are the same as for $x$.

The following result extends the result of Proposition 10 to general lotteries. It relies on the fact that any random variable is distributed as a function of a given normally distributed variable.

Proposition 11 Consider an individual who evaluates through $U_{\delta}$ any lottery $v=\varphi(x)$ where $x$ is normally distributed and whose preferences over the set of all possible lotteries only depend on the distribution of the lottery under consideration. The individual is a CPT agent over the space of all possible lotteries in the sense that her preferences can be represented by the utility function $U_{\delta}$ extended to the space of all possible lotteries and there exists a probability weighting function $g_{\delta}$ such that, for all lottery $v$ with density $f_{v}$ we have $U_{\delta}(v)=\int f_{v, \delta}(s) u(s) d s$ where $g_{\delta}\left(\int_{t}^{\infty} f_{v}(s) d s\right)=\int_{t}^{\infty} f_{v, \delta}(s) d s$.

This corollary provides then the following possible interpretation of CPT: the result of a possibly noisy transmission of the objective distribution to separate (specialized) systems (neural systems or brain areas), the overall evaluation resulting from a social welfare function applied to these systems. The construction of the $Q_{i} \mathrm{~s}$ in the general case is very similar to their construction in the normal case. The resulting global behavior might then be associated intuitively with a possible behavior of the systems that consists in describing any random variable in terms of Gaussian distributions. For instance, a random variable that takes values 0 and 1 with probability $1 / 2$ may be described as a random variable that takes value 1 when a given Gaussian variable $\mathcal{N}(0,1)$ is positive and that takes value 0 when the Gaussian variable is negative. The process $i$ will then transform this binomial distribution into a binomial distribution that is equal to 1 when a Gaussian variable $\mathcal{N}\left(\delta_{i}, 1\right)$ is positive -or equivalently when a Gaussian variable 
$\mathcal{N}(0,1)$ is smaller than $\delta_{i^{-}}$and is equal to 0 when the Gaussian variable $\mathcal{N}\left(\delta_{i}, 1\right)$ is negative.

\subsection{Heterogeneous Beliefs and Ambiguity aversion}

In this section, we show that an individual who evaluates ambiguous lotteries through the social welfare function associated with a collection of agents, each of them being endowed with a plausible probability, may be ambiguity averse.

Let us consider an individual facing a lottery whose payoff $x$ has an ambiguous distribution. The ambiguity of the lottery is characterized by a set of plausible distributions with a set of associated probabilities $\Pi_{x}=\left(Q_{1}^{x}, \ldots, Q_{n}^{x}\right)$. We assume that the agent passes the information to different systems (neural systems or brain areas), each endowed with one of the plausible distributions, in order to evaluate this lottery. We assume that the individual acts like a central planner looking for a Pareto optimal decomposition of the gains/losses from the lottery among the different processes and evaluates the lottery through the social welfare function, i.e.,

$$
U(x)=\max _{\sum x^{i}=x} \sum_{i \in I} E^{Q_{i}^{x}}\left[u\left(x^{i}\right)\right]
$$

We assume that the common utility function is given by $u(x)=\frac{x^{1-\frac{1}{\eta}}}{1-\frac{1}{\eta}}$ with $\eta<1$.

Consider first the classical Ellsberg 3-color urn problem. An urn contains 30 red balls and 60 black and yellow balls in unspecified proportion. A lottery A that pays $\$ 100$ if a red ball is drawn is evaluated at $U(A)=\frac{1}{3} u(100)$. Since the probability for one black ball to be drawn is unknown, and if all possible probabilities of the form $\frac{i}{90}$ for $i=0, \ldots, 60$, are considered as equally plausible, a lottery B that pays $\$ 100$ if a black ball is drawn is evaluated at $U(B)=\left(\frac{1}{61} \sum_{i=0}^{60}\left(\frac{i}{90}\right)^{\eta}\right)^{\frac{1}{\eta}} u(100)$. It is easy to check that $\left(\frac{1}{61} \sum_{i=0}^{60}\left(\frac{i}{90}\right)^{\eta}\right)^{\frac{1}{\eta}}<\frac{1}{3}$ for $\eta<1$ and our individual prefers lottery A to lottery B. Now, the same individual evaluates the lottery C that pays $\$ 100$ if a red or a yellow ball is drawn at $U(C)=\left(\frac{1}{61} \sum_{i=0}^{60}\left(\frac{30+i}{90}\right)^{\eta}\right)^{\frac{1}{\eta}} u(100)$. The lottery $\mathrm{D}$ that pays $\$ 100$ if a black or a yellow ball is drawn is evaluated at $U(D)=\frac{2}{3} u(100)$. 
Such an individual prefers lottery D to lottery C. As in Ellsberg paradox, Savage's independence axiom is violated, even though each process is an expected utility maximizer and satisfies the independence axiom. The individual exhibits ambiguity aversion. In order to generalize this result let us recall the following definitions adapted to our setting from Schmeidler ${ }^{7}$ (1989).

Definition 2 An ambiguous lottery $\left(x,\left(Q_{i}^{x}\right)_{i \in I}\right)$ is defined by a random variable $x$, whose values $(x(\omega))_{\omega \in \Omega}$ represent the set of possible outcomes and by a set $\left(Q_{i}^{x}\right)_{i \in I}$ of plausible probabilities over $\Omega$

Definition 3 If $\left(x,\left(Q_{i}^{1, x}\right)_{i \in I}\right)$ and $\left(x,\left(Q_{i}^{2, x}\right)_{i \in I}\right)$ are two ambiguous lotteries associated to the same random variable $x$, the even mixture of these ambiguous lotteries is the ambiguous lottery $\left(x,\left(\frac{1}{2} Q_{i}^{1, x}+\frac{1}{2} Q_{i}^{2, x}\right)_{i \in I}\right)$.

Definition 4 An agent is called ambiguity averse if she prefers the even mixture of two ambiguous lotteries that she values equally to either of the two lotteries.

Proposition 12 When $\eta<1$, an individual who evaluates ambiguous lotteries through the social welfare function associated to a collection of agents each of them being endowed with one of the plausible probabilities is ambiguity averse.

We have considered as equally plausible all probabilities associated to a given ambiguous lottery. Our results can be generalized to the setting where the individual, based on his subjective information, associates a probability distribution $\left(q_{i}\right)_{i \in I}$ over the set $\left(Q_{i}^{x}\right)_{i \in I}$ where $q_{i}$ $\geq 0$ is the subjective probability that $Q_{i}^{x}$ be the probability associated to the true distribution of the lottery payoffs. In such a setting, the $q_{i}$ s might be integrated in the individual overall utility function as the weights in the social welfare function, i.e.

$$
U(x)=\max _{\sum x^{i}=x} \sum_{i \in I} q_{i} E^{Q_{i}^{x}}\left[u\left(x^{i}\right)\right] .
$$

\footnotetext{
${ }^{7}$ See also Ghirardato (2004)
} 


\subsection{Dynamic setting}

We have until now only considered a two dates setting even though we have considered different possible time horizons for the second date. It is useful to understand how our model might be embedded in a dynamic setting.

Consider as in financial models a diffusion setting. We denote by $W$ a Brownian motion and we assume that $e^{*}$ follows the following stochastic differential equation with constant parameters $d e_{t}^{*}=\left(\mu+\frac{1}{2} \sigma^{2}\right) e_{t}^{*} d t+\sigma e_{t}^{*} d W_{t}$. The distribution of $e_{t}^{*}$ is then, for all $t$, lognormal of the form $\log e_{t}^{*} \sim \mathcal{N}\left(\mu t, \sigma^{2} t\right)$.

Let us first assume as in Section 5.1 that agents' deviation from the objective mean is constant in terms of standard deviation, i.e., that the subjective distributions are of the form $\log \mathcal{N}\left(\mu_{i} t, \sigma^{2} t\right)$ with $\mu_{i} t=\mu t+\delta_{i} \sigma \sqrt{t}$. Following Proposition 10, there exists a probability weighting function $g_{\delta}$ that distorts the objective distribution into the distribution of the group. This function is independent of $t$ and the behavior of the group is then consistent across time.

Let us now consider the case where beliefs heterogeneity results from ambiguity. Different probabilities are considered as plausible and each agent is endowed with one of them. In our diffusion setting, let us consider the simplest case where each probability is defined by its density $d M_{i}=\delta_{i} M_{i} d W$ where the $\delta_{i}$ s are constant. Following Girsanov Theorem, the distribution of $\log e_{t}^{*}$ from agent $i$ point of view is given by $\mathcal{N}\left(\mu_{t}^{i}, \sigma^{2} t\right)$ with $\mu_{t}^{i}=\left(\mu-\frac{1}{2} \sigma^{2}\right) t+\sigma \delta^{i} t$. As seen in Example 1, the ex-ante variance of $\log e_{t}^{*}$ from the representative agent point of view is given by $\left.\operatorname{Var}^{M}\left[\log e_{t}^{*}\right]\right)=\operatorname{Var}\left[\log e_{t}^{*}\right]+\operatorname{Var}_{i}\left(\mu_{t}^{i}\right)=\sigma^{2} t+\sigma^{2} t^{2} \operatorname{Var}_{i} \delta^{i}$. The long term ex-ante variance from the representative agent point of view, is then higher than the objective variance and their ratio converges to infinity.

It is interesting to note that, even though the different agents do not learn and continue to believe in a constant drift $\mu^{i}=\left(\mu-\frac{1}{2} \sigma^{2}\right)+\sigma \delta^{i}$, the group as a whole learns and the drift anticipated by the group converges to the objective drift as long as the objective probability lies in the initial set of plausible probabilities, i.e. as long as we have $\delta_{i}=0$ for some $i$. This point 
can be easily checked in the log utility setting where the representative agent probability is given by $M=\frac{1}{|I|} \sum M_{i}$ and satisfies $d M=\delta_{M} M d W$ with $\delta_{M}=\frac{\sum \delta_{i} M_{i}}{\sum M_{i}}$. By Girsanov Theorem, the date- $t$ drift anticipated by the representative agent is then given by $\mu^{i}=\left(\mu-\frac{1}{2} \sigma^{2}\right)+\sigma \delta_{M}$ which converges almost surely to the objective drift $\left(\mu-\frac{1}{2} \sigma^{2}\right)$.

\section{References}

Abel, A., 2002. An exploration of the effects of pessimism and doubt on asset returns. Journal of Economic Dynamics and Control, 26, 1075-1092.

Abdellaoui, M., 2000. Parameter-free Elicitation of Utilities and Probability Weighting Functions. Management Science 46, 1497-1512.

Brocas, I. and J. Carillo, 2008. The brain as a hierarchical organization. American Economic Review, 98, 1312-46.

Chateauneuf, A. and M. Cohen, 1994. Risk seeking with diminishing marginal utility in a nonexpected utility model. Journal of Risk and Insurance, 9, 77-91.

Cohen, J.D., 2005. The Vulcanization of the Human Brain: a Neural Perspective on Interactions beetween Cognition and Emotion. Journal of Economic Perspectives, Vol. 19, 3-24.

Diecidue, E. and P. Wakker, 2001. On the Intuition of Rank Dependent Utility. Journal of Risk and Insurance, 281-298.

Ghirardato, P., 2004. Defining ambiguity and ambiguity attitude. In Uncertainty in Economic Theory: a collection of essays in honor of David Schmeidler's 65th Birthday. I. Gilboa Editor, Routledge, London.

Gollier, C., 1997. A Note on Portfolio Dominance. Review of Economic Studies, 64, 147-150.

Gollier, C. and R. Zeckhauser, 2005. Aggregation of heterogeneous time preferences. Journal of Political Economy, 113, 4, 878-898.

Gonzalez, R., and G. Wu, 1999. On the shape of the probability weighting function. Cognitive Psychology, 38, 129-166.

Jouini, E., and C. Napp, 2007. Consensus Consumer and Intertemporal Asset pricing with 
Heterogeneous Beliefs. Review of Economic Studies, 2007, 74, 1149-1174.

Jouini, E., and C. Napp, 2008. Discounting and Divergence of Opinion. Forthcoming, Journal of Economic Theory.

Landsberger, M., and I. Meilijson, 1990. Demand for risky assets: a portfolio analysis. Journal of Economic Theory, 50, 204-213.

Landsberger, M., and I. Meilijson, 1993. Mean Preserving Portfolio Dominance. The Review of Economic Studies, 60, 475-485.

Lengwiler, Y., 2005. Heterogeneous patience and the term structure of real interest rates. American Economic Review, 95, 890-896.

Loewenstein G., and D. Prelec, 1992. Anomalies in intertemporal choices: evidence and an interpretation. The Quarterly Journal of Economics, 107, 573-597.

Lopes, L., 1987. Between hope and fear: the psychology of risk. Advances in Experimental Social Psychology, 20, 255-295.

Nocetti, D., Jouini, E., and C. Napp, 2008. Properties of the social discount rate in a Benthamite framework with heterogeneous degrees of impatience. Management Science, 54, $1822-1826$.

Prelec, D., 1998. The Probability Weighting Function. Econometrica, 66, 497-527.

Reinschmidt, K.F., 2002. Aggregate Social Discount Rate derived from individual discount rates. Management Science, 48, 307-312.

Schmeidler, D., 1989. Subjective Probability and Expected Utility without Additivity. Econometrica, 57, 571-587.

Shefrin, H., 2005. A Behavioral Approach to Asset Pricing. Elsevier.

Shefrin, H. and R. Thaler, 1988. The Behavioral Life-Cycle Hypothesis. Economic Inquiry, $26,609-43$.

Thaler, R. and H. Shefrin, 1981. An Economic Theory of Self-Control. Journal of Political Economy, 89, 392-406. 
Tversky, A., and C.R. Fox, 1995. Ambiguity Aversion and Comparative Ignorance. Quarterly Journal of Economics, 110, 585-603.

Tversky, A., and D. Kahneman, 1992. Advances in Prospect Theory: Cumulative Representation of Uncertainty. Journal of Risk and Uncertainty, 5, 297-323.

Yaari, 1987. The Dual Theory of Choice under Risk. Econometrica, 55, 95-115. 


\section{Proof of Proposition 1}

At the Pareto optimum, we have

$$
\lambda_{i} M^{i} u^{\prime}\left(y^{i}\right)=q
$$

for some random variable $q$. It follows that

$$
y^{i}=\left[\frac{q}{\lambda_{i} M^{i}}\right]^{-\eta}
$$

hence

$$
e^{*}=\sum_{i \in I}\left[\frac{q}{\lambda_{i} M^{i}}\right]^{-\eta}=q^{-\eta} \sum_{i \in I}\left[\frac{1}{\lambda_{i} M^{i}}\right]^{-\eta}
$$

and

$$
y^{i}=e^{*} \frac{\left[\lambda_{i} M^{i}\right]^{\eta}}{\sum_{i \in I}\left[\lambda_{i} M^{i}\right]^{\eta}} .
$$

We have then

$$
\begin{aligned}
\sum_{i \in I} \lambda_{i} E\left[M^{i} u\left(y^{i}\right)\right] & =\sum_{i \in I} \lambda_{i} E\left[M^{i} \frac{\left[\lambda_{i} M^{i}\right]^{\eta-1}}{\left(\sum_{i \in I}\left[\lambda_{i} M^{i}\right]^{\eta}\right)^{1-\frac{1}{\eta}}} u\left(e^{*}\right)\right] \\
& =E\left[\frac{\sum_{i \in I}\left[\lambda_{i} M^{i}\right]^{\eta}}{\left(\sum_{i \in I}\left[\lambda_{i} M^{i}\right]^{\eta}\right)^{1-\frac{1}{\eta}}} u\left(e^{*}\right)\right] \\
& =E\left[\left[\sum_{i \in I}\left[\lambda_{i} M^{i}\right]^{\eta}\right]^{1 / \eta} u\left(e^{*}\right)\right]
\end{aligned}
$$

and $U\left(e^{*}\right)=E\left[M u\left(e^{*}\right)\right]$ with $M=\left[\sum_{i \in I} \lambda_{i}^{\eta}\left(M^{i}\right)^{\eta}\right]^{1 / \eta}$.

\section{Proof of Corollary 2}


We have

$$
\begin{aligned}
E\left[M h\left(e^{*}\right)\right] & =E\left[\left(\sum_{i \in I} \gamma_{i}\left(M^{i}\right)^{\eta}\right)^{1 / \eta} h\left(e^{*}\right)\right] \\
& =E\left[\left(\sum_{i \in I} \gamma_{i}\left(\frac{f^{i}}{f}\left(e^{*}\right)\right)^{\eta}\right)^{1 / \eta} h\left(e^{*}\right)\right] \\
& =E\left[\frac{\left(\sum_{i \in I} \gamma_{i}\left(f^{i}\left(e^{*}\right)\right)^{\eta}\right)^{1 / \eta}}{f\left(e^{*}\right)} h\left(e^{*}\right)\right] \\
& =\int \frac{\left(\sum_{i \in I} \gamma_{i}\left(f^{i}(x)\right)^{\eta}\right)^{1 / \eta}}{f(x)} h(x) f(x) d x \\
& =\int\left(\sum_{i \in I} \gamma_{i}\left(f^{i}(x)\right)^{\eta}\right)^{1 / \eta} h(x) d x
\end{aligned}
$$

hence $f^{M}=\left(\sum_{i \in I} \gamma_{i}\left(f_{i}\right)^{\eta}\right)^{1 / \eta}$.

\section{Proofs for Example 2}

1. Proof that the distribution of $\log e^{*}$ is bimodal for $\left|\mu_{1}-\mu_{2}\right|>2 \sigma / \sqrt{\eta}$ and unimodal for $\left|\mu_{1}-\mu_{2}\right| \leq 2 \sigma / \sqrt{\eta}$. We have

$$
\begin{aligned}
\left(f^{\log }\right)^{\eta}= & \frac{1}{2}\left(f_{1}^{\log }\right)^{\eta}+\frac{1}{2}\left(f_{2}^{\log }\right)^{\eta}=\frac{1}{2 \sqrt{2 \pi} \sigma} \exp \left(-\frac{\eta\left(x-\mu_{1}\right)^{2}}{2 \sigma^{2}}\right) \\
& +\frac{1}{2 \sqrt{2 \pi} \sigma} \exp \left(-\frac{\eta\left(x-\mu_{2}\right)^{2}}{2 \sigma^{2}}\right) .
\end{aligned}
$$

This function has either two maxima that are symmetric with respect to $\frac{\mu_{1}+\mu_{2}}{2}$ or only one maximum at $\frac{\mu_{1}+\mu_{2}}{2}$. In the first case $\frac{\mu_{1}+\mu_{2}}{2}$ would be a local minimum. It suffices then to analyse the sign of the second derivative of $\left(f^{\log }\right)^{\eta}$ at $\frac{\mu_{1}+\mu_{2}}{2}$. We obtain that the distribution is bimodal for $\left|\mu_{1}-\mu_{2}\right|>2 \sigma / \sqrt{\eta}$ and unimodal for $\left|\mu_{1}-\mu_{2}\right| \leq 2 \sigma / \sqrt{\eta}$.

2. Proof that for $\mu=\frac{\mu_{1}+\mu_{2}}{2}$ the distribution of $\log e^{*}$ is portfolio dominated by the objective distribution. The ratio between the density of $\log e^{*}$ under $Q$ and the 
density of $\log e^{*}$ under $P$ is given by

$\frac{f^{M \log }}{f^{\log }}(x)=\left(\frac{1}{2} \exp \left(\eta \frac{-2(x-\mu)\left(\mu-\mu_{1}\right)+\mu^{2}-\mu_{1}^{2}}{2 \sigma^{2}}\right)+\frac{1}{2} \exp \left(\eta \frac{-2 x\left(\mu-\mu_{2}\right)+\mu^{2}-\mu_{2}^{2}}{2 \sigma^{2}}\right)\right)^{\frac{1}{\eta}}$

which is clearly symmetric with respect to $\mu$, decreasing before $\mu$ and increasing after $\mu$. Moreover, since the distributions of $\log e^{*}$ under $Q$ and under $P$ are both symmetric with respect to $\mu$, we have $E^{Q}\left[\log e^{*}\right]=E^{P}\left[\log e^{*}\right]=\mu$. These properties give $\operatorname{Var}^{Q}\left[\log e^{*}\right]>$ $\operatorname{Var}^{P}\left[\log e^{*}\right]$ (see Jouini and Napp, 2008).

3. Proof that for general $\left(\mu_{i}\right), \operatorname{Var}^{Q}\left[\log e^{*}\right]>\operatorname{Var}^{P}\left[\log e^{*}\right]$. For general $\left(\mu_{i}\right), f^{M \log }$ is symmetric with respect to $\frac{\mu_{1}+\mu_{2}}{2}$ which gives $E^{Q}\left[\log e^{*}\right]=\frac{\mu_{1}+\mu_{2}}{2}$. Furthermore, we may apply the same reasoning as in 2. to compare the distribution of $\log e^{*}$ under $Q$ with the distribution whose density is given by $\frac{1}{\sqrt{2 \pi} \sigma} \exp -\frac{\left(x-\frac{\mu_{1}+\mu_{2}}{2}\right)^{2}}{2 \sigma^{2}}$. We then have $\operatorname{Var}^{Q}\left[\log e^{*}\right]>\sigma^{2}=\operatorname{Var}^{P}\left[\log e^{*}\right]$

\section{Proof that a higher level of risk tolerance induces a Portfolio Dominated shift}

in the representative agent distribution. For two different values $\eta$ and $\eta^{\prime}$ of the risk tolerance parameter, it suffices to consider $\frac{f_{\eta^{\prime}}^{M \log }}{f_{\eta}^{M \log }}$ and to apply the same reasoning as in 2 .

\section{Proof of Proposition 3}

1. If $\lim _{\infty} \frac{f_{\text {opt }}}{f}=\lim _{-\infty} \frac{f_{\text {pess }}}{f}=\infty$ and $\lim _{-\infty} \frac{f_{\text {opt }}}{f}=\lim _{\infty} \frac{f_{\text {pess }}}{f}=0$ then the representative agent density function is such that $\lim _{-\infty} \frac{f_{M}}{f}=\lim _{\infty} \frac{f_{M}}{f}=\infty$ and $\frac{f_{M}}{f}$ can not be monotone.

2. This is immediate according to $\lim _{-\infty} \frac{f_{M}}{f}=\lim _{\infty} \frac{f_{M}}{f}=\infty$.

3. It suffices to remark that $f^{M}=f_{\text {opt }}^{\max }\left(\gamma_{\text {opt }}^{\max }+\sum_{\substack{i=1, \ldots, N \\ i \neq o p t}}\left(\frac{f_{i}}{f_{\text {opt }}^{\max }}\right)^{\eta}\right)^{1 / \eta}$. If $\frac{f_{i}}{f_{\text {opt }}^{\text {max }}}$ is nonincreasing for all $i$ then $\left(\gamma_{\text {opt }}^{\max }+\sum_{\substack{i=1, \ldots, N \\ i \neq o p t}}\left(\frac{f_{i}}{f_{\text {opt }}^{\text {max }}}\right)^{\eta}\right)^{1 / \eta}$ is bounded away from 0 and $\infty$ in 
the neighborhood of $\infty$ and we have $f^{M} \sim_{\infty} f_{\text {opt }}^{\max }$. The result at the neighborhood of $-\infty$ is obtained similarly.

\section{Proof of Proposition 4}

1. Let $g$ be given by $\int_{u}^{\infty} f_{M}(x) d x=g\left[\int_{u}^{\infty} f(x) d x\right]$. We have $f_{M}(x)=g^{\prime}\left[\int_{u}^{\infty} f(x) d x\right] f(u)$ and $g^{\prime}\left[\int_{u}^{\infty} f(x) d x\right]=\frac{f_{M}}{f}(u)$. We also have $-f(u) g^{\prime \prime}\left[\int_{u}^{\infty} f(x) d x\right]=\left(\frac{f_{M}}{f}\right)^{\prime}(u)$ which gives that the concavity of $g$ is governed by the sign of $\left(\frac{f_{M}}{f}\right)^{\prime}$. Remark that $\left(\frac{f_{M}}{f}\right)^{\prime}$ is negative in a neighborhood of $-\infty$ and then that $g^{\prime \prime}$ is positive and $g$ is convex in a neighborhood of 1 . Similarly, we have that $\left(\frac{f_{M}}{f}\right)^{\prime}$ is positive in a neighborhood of $\infty$ and then that $g^{\prime \prime}$ is negative and $g$ is concave in a neighborhood of 1 . Finally, $\left(\frac{f_{M}}{f}\right)^{\prime}$ is a combination of exponentials where the decreasing exponentials have a negative weight and the increasing exponentials have a positive weight. The function $\left(\frac{f_{M}}{f}\right)^{\prime}$ is then increasing. The function $g$ is then inverse S-shaped: concave then convex.

2. Since $g^{\prime}\left[\int_{u}^{\infty} f(x) d x\right]=\frac{f_{M}}{f}(u)$, we have $g^{\prime}(0)=\frac{f_{M}}{f}(\infty)=\infty$. If $g^{\prime \prime}(0)$ is well defined, we have $g^{\prime \prime}(0)<0$ and hence $g^{\prime \prime}(x)<0$ in a neighborhood of 0 . The probability weighting function is then concave for small probabilities. The result in the neighborhood of 1 is obtained similarly.

\section{Proof of Proposition 5}

1. Let us consider a distribution of the means that is described by a density function $h$. The associated representative agent cumulative distribution function is given by $\frac{1}{\sqrt{2 \pi \sigma^{2}}} \int d h(\mu) \int_{-\infty}^{x} \exp -\frac{(s-\mu)^{2}}{2 \sigma^{2}} d s$. Since the function $\mu \rightarrow \int_{-\infty}^{x} \exp -\frac{(s-\mu)^{2}}{2 \sigma^{2}} d s$ is decreasing a FSD shift of $h$ decreases the value of $\int d h(\mu) \int_{-\infty}^{x} \exp -\frac{(s-\mu)^{2}}{2 \sigma^{2}} d s$ and leads then to a FSD dominating distribution function for the representative agent. 
2. Let us consider a distribution $\left(\gamma_{i}^{\prime}\right)$ and a FSD dominated shift $\left(\gamma_{i}\right)$. We want to prove that $\sum \gamma_{i}^{\prime} F_{i} \geq \sum \gamma_{i} F_{i}$. For a given $x$, letting $x_{i}$ denote the quantity $F_{i}(x)$, it suffices to prove that $\sum \gamma_{i}^{\prime} x_{i} \geq \sum \gamma_{i} x_{i}$ for a nondecreasing family $\left(x_{i}\right)_{i \in I}$ which is true since $\left(\gamma_{i}^{\prime}\right)$ dominates $\left(\gamma_{i}\right)$ in the sense of the FSD.

3. Let us consider a distribution $\left(\gamma_{i}^{\prime}\right)$ and a MLR dominated shift $\left(\gamma_{i}\right)$. It suffices to prove that $\frac{\left(\sum \gamma_{i}^{\prime} f_{i}^{\eta}\right)^{\frac{1}{\eta}}}{\left(\sum \gamma_{i} f_{i}^{\eta}\right)^{\frac{1}{\eta}}}$ is increasing or that $\frac{\sum \gamma_{i}^{\prime} G_{i}}{\sum \gamma_{i} G_{i}}$ is increasing with $G_{i}=f_{i}^{\eta}$. Without any loss of generality, we may assume that all the considered functions are differentiable and let us consider the derivative of $\frac{\sum \gamma_{i}^{\prime} G_{i}}{\sum \gamma_{i} G_{i}}$

$$
\begin{aligned}
& \left(\frac{\sum \gamma_{i}^{\prime} G_{i}}{\sum \gamma_{i} G_{i}}\right)^{\prime}=\frac{\left(\sum \gamma_{i}^{\prime} G_{i}^{\prime}\right)\left(\sum \gamma_{i} G_{i}\right)-\left(\sum \gamma_{i}^{\prime} G_{i}\right)\left(\sum \gamma_{i} G_{i}^{\prime}\right)}{\left(\sum \gamma_{i} G_{i}\right)^{2}} \\
& =\frac{\sum_{f_{i} \succeq f_{j}} \gamma_{i} \gamma_{j}\left(\frac{\gamma_{i}^{\prime}}{\gamma_{i}}-\frac{\gamma_{j}^{\prime}}{\gamma_{j}}\right)\left(G_{i}^{\prime} G_{j}-G_{i} G_{j}^{\prime}\right)}{\left(\sum \gamma_{i} G_{i}\right)^{2}} .
\end{aligned}
$$

Remark that for $f_{i} \succeq f_{j}$ we have $G_{i} \succeq G_{j}$ and then $G_{i}^{\prime} G_{j}-G_{i} G_{j}^{\prime} \geq 0$. Furthermore, for $f_{i} \succeq f_{j}$ we also have $\frac{\gamma_{i}^{\prime}}{\gamma_{i}}-\frac{\gamma_{j}^{\prime}}{\gamma_{j}} \geq 0$ which leads to the conclusion.

\section{Proof of Proposition 6}

It is immediate that $\mu_{1}, \mu_{2}$, and $\mu_{3}$ can be written in the form $\mu_{2}-h, \mu_{2}, \mu_{2}+h$ for some $h>0$. For the distribution of individual characteristics $\left(\gamma_{i}\right)$, the representative agent distribution function is given by $\frac{1}{\sqrt{2 \pi \sigma^{2}}} \sum_{i} \gamma_{i} \int_{-\infty}^{x} \exp -\frac{\left(s-\mu_{i}\right)^{2}}{2 \sigma^{2}} d s$. The symmetric mean preserving spread induces a modification of this distribution that is positively proportional to $\frac{1}{\sqrt{2 \pi \sigma^{2}}}\left(\int_{-\infty}^{x} \exp \left(-\frac{\left(s-\mu_{2}+h\right)^{2}}{2 \sigma^{2}}\right)-2 \exp \left(-\frac{\left(s-\mu_{2}\right)^{2}}{2 \sigma^{2}}\right)+\exp \left(-\frac{\left(s-\mu_{2}-h\right)^{2}}{2 \sigma^{2}}\right)\right) d s$. Simple computations permit to show that this modification is positive for $x \leq \mu_{2}$ and negative for $x \geq \mu_{2}$. A symmetric mean preserving spread leads then to a distribution function that is above (resp. below) the original distribution function below a given threshold. We have then an increase of the level of discriminability.

\section{Proof of Proposition 7}


Replacing $M^{i}$ by $M^{i} D^{i}$ in the proof of Proposition 1 , we easily get that

$$
U\left(e_{t}^{*}\right)=\left[\sum_{i \in I}\left[\lambda_{i} M_{t}^{i} D_{t}^{i}\right]^{\eta}\right]^{1 / \eta}
$$

Now, if the characteristics $\left(\lambda_{i}, M_{t}^{i}, D_{t}^{i}\right)$ are independent, then

$$
\left[\sum_{i \in I}\left[\lambda_{i} M_{t}^{i} D_{t}^{i}\right]^{\eta}\right]^{1 / \eta}=\left[\left(\frac{1}{|I|} \sum_{i \in I}\left(M_{t}^{i}\right)^{\eta}\right)\right]^{1 / \eta}\left[\left(\frac{1}{|I|} \sum_{i \in I}\left(D_{t}^{i}\right)^{\eta}\right)\right]^{1 / \eta}
$$

and

$$
\sum_{i \in I} \lambda_{i} E\left[M_{t}^{i} D_{t}^{i} u\left(y_{t}^{i}\right)\right]=E\left[\left(\frac{1}{|I|} \sum_{i \in I}\left(M_{t}^{i}\right)^{\eta}\right)^{1 / \eta}\left(\frac{1}{|I|} \sum_{i \in I}\left(D_{t}^{i}\right)^{\eta}\right)^{1 / \eta} u\left(e_{t}^{*}\right)\right]
$$

\section{Proof of Proposition 8}

We prove the proposition for $\rho_{m}^{D}$ since it is easy to check that all the derived properties are inherited by $\rho_{a}^{D}(t)=\frac{1}{t} \int_{0}^{t} \rho_{m}^{D}(s) d s$.

1. Immediate.

2. The representative agent time preference rate $\rho_{m}^{D}(t)=\sum_{i=1}^{N} \frac{\exp \left(-\eta \rho_{i} t\right)}{\sum_{i=1}^{N} \exp \left(-\eta \rho_{i} t\right)} \rho_{i}$ is an average of the $\rho_{i}$ s with weights that decrease with $\rho_{i}$. Such an average is smaller than the equally weighted average.

3. Denote $\theta_{i}=\exp \left(-\eta \rho_{i} t\right)$. We have $\frac{d \rho_{m}^{D}(t)}{d t}=-\left(\frac{\sum_{i \in I} \theta_{i} \rho_{i}^{2}}{\sum_{i \in I} \theta_{i}}-\left(\frac{\sum_{i \in I} \theta_{i} \rho_{i}}{\sum_{i \in I} \theta_{i}}\right)^{2}\right)$ which is negative.

We have $\rho_{m}^{D}(t)=\frac{\rho_{\text {inf }}+\sum_{i \neq \text { inf }}^{N} \exp \left(-\eta\left(\rho_{i}-\rho_{\text {inf }}\right) t\right) \rho_{i}}{1+\sum_{i \neq \text { inf }}^{N} \exp \left(-\eta\left(\rho_{i}-\rho_{\text {inf }}\right) t\right)}$ and $\exp \left(-\eta\left(\rho_{i}-\rho_{\text {inf }}\right) t\right) \rho_{i} \rightarrow \infty 0$ we have then $\rho_{m}^{D}(t) \rightarrow \rho_{\text {inf }}$ 
The proof of 1 . is inspired from Jouini and Napp (2008) and the proof of 2. is inspired from Nocetti et al. (2008).

1. We have

$$
\rho_{a}^{D}(t) \equiv-\frac{1}{\eta t} \ln E[\exp (-\eta \rho t)]
$$

where $E$ is the expectation operator associated with the distribution of $\left(\rho_{i}\right)$. For a given $t$, the function $\rho \rightarrow \exp (-\eta \rho t)$ is decreasing (and convex) and, by definition, a FSD (resp. SSD) shift in the distribution of $\left(\rho_{i}\right)$ decreases the value of $E[\exp (-\eta \rho t)]$ and increases $\rho_{a}^{D}(t)$.

2. We have then

$$
\rho_{m}^{D}(t)=\frac{E[\rho \exp (-\eta \rho t)]}{E[\exp (-\eta \rho t)]}
$$

where $E$ is the expectation operator associated with the distribution of $\left(\rho_{i}\right)$.

Let us now consider $P^{1}$ and $P^{2}$, two distributions such that $P^{2} \succeq_{M L R} P^{1}$. By definition, the density $\phi=\frac{d P^{2}}{d P^{1}}$ is nondecreasing in $\rho$ (in other words $i \rightarrow \phi^{i}$ and $i \rightarrow \rho_{i}$ are comonotonic). We have then, $\frac{E^{P^{2}}[\rho \exp (-\eta \rho t)]}{E^{P^{2}}[\exp (-\eta \rho t)]}=\frac{E^{P^{1}}[\phi \rho \exp (-\eta \rho t)]}{E^{P^{1}}[\phi \exp (-\eta \rho t)]}=\frac{E^{Q}[\phi \rho]}{E^{Q}[\phi]}$ where $Q$ is defined by a density with respect to $P^{1}$ equal (up to a constant) to $\exp (-\eta \rho t)$. Since $\phi$ is nondecreasing in $\rho$, we have

$$
E^{Q}[\phi \rho] \geq E^{Q}[\phi] E^{Q}[\rho],
$$

hence

$$
\begin{aligned}
& \frac{E^{P^{2}}[\rho \exp (-\eta \rho t)]}{E^{P^{2}}[\exp (-\eta \rho t)]} \geq E^{Q}[\rho], \\
& \geq \frac{E^{P^{1}}[\rho \exp (-\eta \rho t)]}{E^{P^{1}}[\exp (-\eta \rho t)]} .
\end{aligned}
$$

Let us assume now that $P^{2} \succeq_{P D} P^{1}$ and let us consider $\rho_{m}^{D, P^{2}}(t)$ and $\rho_{m}^{D, P^{1}}(t)$ the 
associated representative agent time preference rates. We have $\rho_{m}^{D, P^{2}}(t)=\frac{E^{P^{2}}[\rho \exp (-\eta \rho t)]}{E^{P^{2}}[\exp (-\eta \rho t)]}$ and then $E^{P^{2}}\left[u^{\prime}(\rho)\left(\rho-\rho_{m}^{D, P^{2}}\right)\right]=0$ with $u(\rho)=-\exp (-\eta \rho t)$. By definition, this implies $E^{P^{1}}\left[u^{\prime}(\rho)\left(\rho-\rho_{m}^{D, P^{2}}\right)\right] \leq 0$ hence $\rho_{m}^{D, P^{2}} \geq \rho_{m}^{D, P^{1}}$. 
Proof of Proposition 10 1. Let $x \in X$ with $x \sim \mathcal{N}\left(\mu, \sigma^{2}\right)$. We have $x \sim_{Q_{i}} \mathcal{N}\left(\mu+\delta_{i} \sigma, \sigma^{2}\right)$ From Proposition 1, there exists $Q$ such that $U_{\delta}(x)=E^{Q}[x]$ and the density of $x$ under $Q$ is given by $f_{x, \delta}(s)=\left[\frac{1}{n} \sum_{i=1}^{N}\left(f_{x, \delta_{i}}\right)^{\eta}\right]^{1 / \eta}$ where $f_{x, \delta_{i}}$ is the density of $x$ under $Q_{i}$. We then have $U_{\delta}(x)=\int f_{x, \delta}(s) u(s) d s$. It suffices to prove that $\int_{t}^{\infty} f_{x, \delta}(s) d s$ is a function of $\int_{t}^{\infty} f_{x}(s) d s$ that does not depend on $x$, i.e. that does not depend upon $\mu$ and $\sigma$. Let $g_{\delta}$ be the function defined by $g_{\delta}\left(\frac{1}{\sqrt{2 \pi}} \int_{t}^{\infty} \exp \left(-\frac{x^{2}}{2}\right) d s\right)=\int_{t}^{\infty}\left[\frac{1}{n} \sum_{i=1}^{N}\left(\frac{1}{\sqrt{2 \pi}} \exp \left(-\eta \frac{\left(x-\delta_{i}\right)^{2}}{2}\right)\right)\right]^{1 / \eta} d s$ for all $t$. The function $g_{\delta}$ is completely defined on $[0,1]$ and by a simple change of variables, we have $g_{\delta}\left(\frac{1}{\sqrt{2 \pi} \sigma} \int_{t}^{\infty} \exp \left(-\frac{(x-\mu)^{2}}{2 \sigma^{2}}\right) d s\right)=\int_{t}^{\infty}\left[\frac{1}{n} \sum_{i=1}^{N}\left(\frac{1}{\sqrt{2 \pi} \sigma} \exp \left(-\eta \frac{\left(x-\delta_{i} \sigma-\mu\right)^{2}}{2 \sigma^{2}}\right)\right)\right]^{1 / \eta} d s$ for all $t$ and we then have $\int_{t}^{\infty} f_{x, \delta}(s) d s=g_{\delta}\left(\int_{t}^{\infty} f_{x}(s) d s\right)$.

2. The function $g_{\delta}$ is the same as in Proposition 4.

3. Direct application of Proposition 5.

\section{Proof of Proposition 11}

Let $v$ such that $v=\varphi(x)$ where $x$ is normally distributed. By definition, we have $U_{\delta}(v)=$ $\sup _{\sum v_{i}} E^{Q_{i}}\left[u\left(v_{i}\right)\right]$. We denote by $f_{v}^{i}$ the density of $v$ with respect to $Q^{i}$. By Proposition 1 and Corollary 2, we have $U_{\delta}(v)=\int f_{v, \delta}(s) u(s) d s$ with $f_{v, \delta}=\left(\sum\left(f_{v}^{i}\right)^{\eta}\right)^{\frac{1}{\eta}}$. We clearly have $f_{v, \delta}=$ $\varphi^{\prime} f_{x, \delta} \circ \varphi$ and $f_{v}^{i}=\varphi^{\prime} f_{x}^{i} \circ \varphi$ and since $\int_{t}^{\infty} f_{x, \delta}(s) d s=\int_{t}^{\infty}\left(\sum\left(f_{x}^{i}\right)^{\eta}\right)^{\frac{1}{\eta}}(s) d s=g_{\delta}\left(\int_{t}^{\infty} f_{x}(s) d s\right)$, a simple change of variable leads to $\int_{t}^{\infty} f_{v, \delta}(s) d s=g_{\delta}\left(\int_{t}^{\infty} f_{v}(s) d s\right)$. We have then the result over the set of transformations of normal distributions.

Let us consider now a random variable $v$ and a normally distributed random variable $x$. We know that $v$ has the same distribution as $F_{v}^{-1}\left[F_{x}(x)\right]$ where $F_{v}^{-1}(p)$ is defined by $F_{v}^{-1}(p)=$ $\inf \left\{t: F_{v}(t) \geq p\right\}$. If the individual has preferences that only depend on the distribution, it suffices to set $U_{\delta}(v)=U_{\delta}(\varphi(x))$ with $\varphi=F_{v}^{-1} \circ F_{x}$ which is perfectly defined. We then have $U_{\delta}(x)=\int_{t}^{\infty} f_{\varphi(x), \delta}(s) u(s) d s$ with $\int_{t}^{\infty} f_{\varphi(x), \delta}(s) d s=g_{\delta}\left(\int_{t}^{\infty} f_{\varphi(x)}(s) d s\right)$ and since $v$ is distributed like $\varphi(x)$, we have $\int_{t}^{\infty} f_{\varphi(x), \delta}(s) d s=g_{\delta}\left(\int_{t}^{\infty} f_{v}(s) d s\right)$, and the result follows. 
Let us consider $\left(x,\left(Q_{i}^{1, x}\right)_{i \in I}\right)$ and $\left(x,\left(Q_{i}^{2, x}\right)_{i \in I}\right)$ two lotteries that our agent values equally. For all $i$ in $I$, let us denote by $M_{i}^{1}$ and $M_{i}^{2}$ the densities respectively associated to $Q_{i}^{1, x}$ and $Q_{i}^{2, x}$. We have $E\left[\left(\sum_{i \in I} M_{i}^{\eta}\right)^{\frac{1}{\eta}} u(x)\right]=E\left[\left(\sum_{i \in I} N_{i}^{\eta}\right)^{\frac{1}{\eta}} u(x)\right]$. For $\eta \leq 1$, we have $\left(\sum_{i \in I}\left(\frac{1}{2} M_{i}+\frac{1}{2} N_{i}\right)^{\eta}\right)^{\frac{1}{\eta}} \geq$ $\frac{1}{2}\left(\sum_{i \in I}\left(M_{i}^{\eta}\right)\right)^{\frac{1}{\eta}}+\frac{1}{2}\left(\sum_{i \in I}\left(N_{i}^{\eta}\right)\right)^{\frac{1}{\eta}}$ which gives $E\left[\left(\sum_{i \in I}\left(\frac{1}{2} M_{i}+\frac{1}{2} N_{i}\right)^{\eta}\right)^{\frac{1}{\eta}} u(x)\right] \geq E\left[\left(\sum_{i \in I} M_{i}^{\eta}\right)^{\frac{1}{\eta}} u(x)\right]$. 


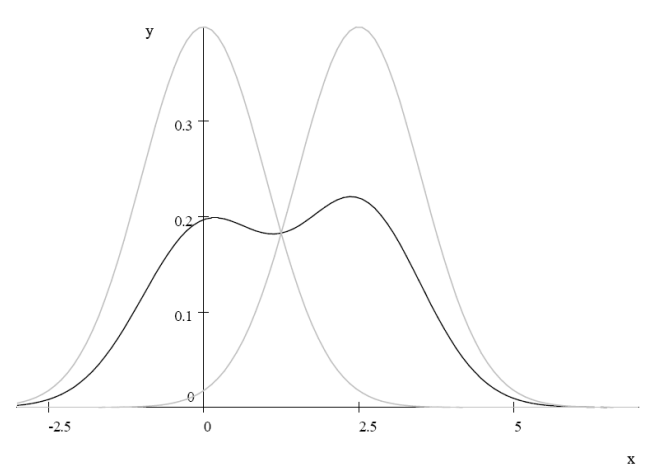

Figure 1: In this figure, we have represented in black the consensus belief in a log-utility agents setting. A proportion of $47 \%$ of the agents believe that $\log e \sim \mathcal{N}(0,1)$ and the remaining $53 \%$ believe that $\log e \sim \mathcal{N}(2.5,1)$. The beliefs of these two categories of agents are represented in grey.

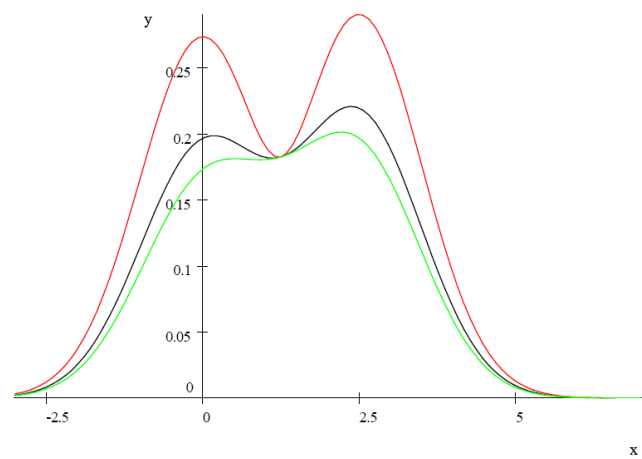

Figure 2: In this figure we represent the consensus belief for 3 different levels of risk aversion. We assume that a proportion of $47 \%$ of the agents believe that $\log e \sim \mathcal{N}(0,1)$ and the remaining $53 \%$ believe that $\log e \sim \mathcal{N}(2.5,1)$. The upper curve corresponds to $\eta=2$, the lower curve to $\eta=0.8$ and the middle curve to $\eta=1$. An increase of $\eta$ increases the distance between the peaks and their size.

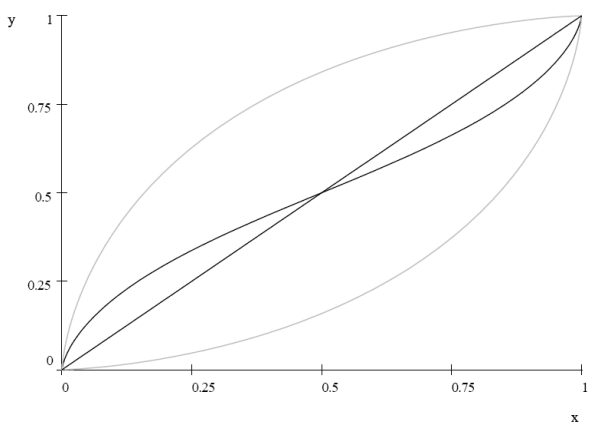

Figure 3: In this figure we represent in black the representative agent probability weighting function in a model with two logarithmic utility agents. One of them overestimates the objective mean by one standard deviation and the other one underestimates it by one standard deviation. We also represent in grey the individual probability weighting functions (the concave one corresponds to the optimistic agent). 


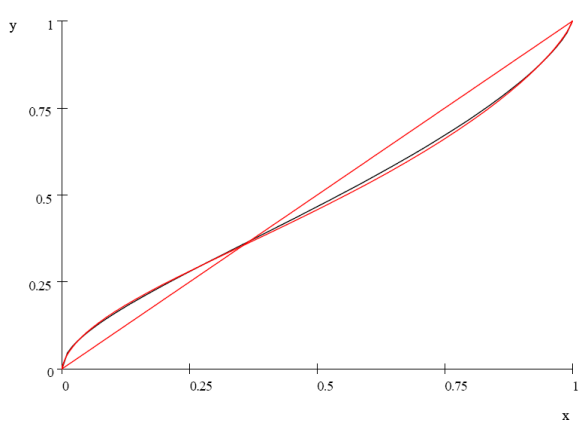

Figure 4: In this figure we represent Prelec's function $\exp \left(-(-\ln p)^{\gamma}\right)$ with $\gamma=0.73$ that corresponds to a standard specification. We also represent the probability weighting function corresponding to a model with two log-utility agents. The first one underestimates the objective average by $120 \%$ of the standard deviation and has a weight of $30 \%$. The second one overestimates the objective average by $60 \%$ of the standard deviation and has a weight of $70 \%$.

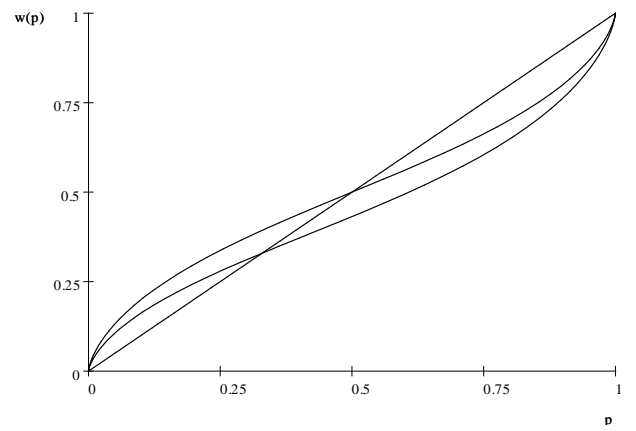

Figure 5: In this figure we represent the probability weighting function of the representative agent in a model with logarithmic utility agents. In the upper curve curve the optimistic and the pessimistic agents are equally weighted. In the lower curve, the pessimistic agents have a $60 \%$ weight and the optimistic ones have a $40 \%$ weight. Attractiveness decreases with the weight granted to the pessimistic agents.

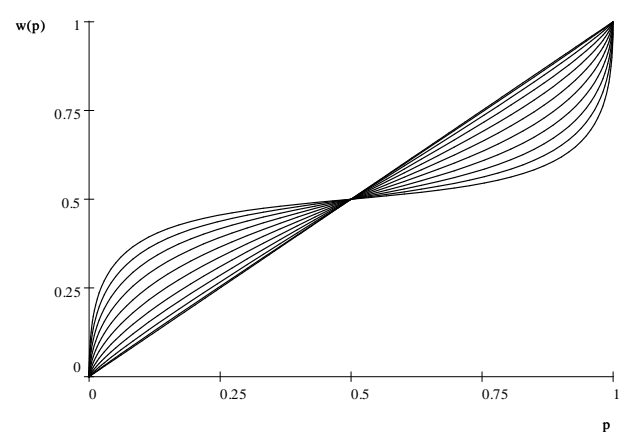

Figure 6: The probability weighting function for different levels of divergence of belief. Both agents agree on a normal distribution but one of them overestimates the objective mean by $\delta$ times the standard deviation while the other one underestimates it by $\delta$ times the standard deviation. The value of $\delta$ ranges from 0 to 2. The discriminability decreases with $\delta$ (in other words the curvature increases with $\delta$ ). 